\title{
The Role of TiN Precipitation in Determining Microporosity in Castings of IN718 Alloy
}

\author{
A. Mitchell and A.J. Schmalz \\ Department of Metals and Materials Engineering \\ University of British Columbia, Vancouver, Canada
}

\begin{abstract}
The various specifications for IN718 to be used in castings have developed from experience over the years in regard to the nitrogen content necessary to avoid excessive microporosity. The presence of this porosity can lead to problems in mechanical properties, particularly in elongation and stress rupture life at high temperature, due to a void coalescence mechanism.

The nitrogen is precipitated as $\mathrm{TiN}$, or as a $\mathrm{Ti} / \mathrm{Nb}$ carbonitride, depending on the temperature and composition of the alloy grade, and at the eutectic temperature essentially all of the nitrogen in the alloy is present as a solid particulate precipitate. The particles form in the interdendritic liquid and act as a blocking agent for the flow necessary to fill the micro-shrinkage as it develops in the last stages of solidification.

In this work we present the results of a study to determine the relation between the amount of precipitate present at various stages in the solidification and the quantity of microporosity present in the solid casting. The castings were made by conventional vacuum investment DS techniques, using alloys with varying compositions to change the nitride precipitation temperature and the eutectic quantity. The dendrite spacing was controlled through the gradient/rate conditions.

It was found that there is a relation between the nitrogen content and microporosity, but that it is complex and depends on the dendrite network structure as well as details of the eutectic freezing process. The present specifications for nitrogen content in commercial alloy are found to be essentially correct, but should be used in conjunction with a knowledge of the casting microstructure and solidification conditions in order to avoid property problems in the finished part.
\end{abstract}

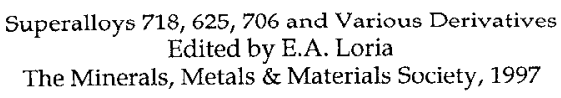

